\title{
Avaliação da qualidade de vida de cafeicultores de Poço Fundo-MG com o instrumento SF-36 durante a pandemia do COVID-19
}

\author{
Evaluation of the quality of life of coffee growers in Poço Fundo-MG with the SF-36 instrument \\ during the COVID-19 pandemic \\ Evaluación de la calidad de vida de los cafetaleros de Poço Fundo-MG con el instrumento SF-36 \\ durante la pandemia COVID-19
}

Recebido: 18/11/2021 | Revisado: 24/11/2021 | Aceito: 25/11/2021 | Publicado: 05/12/2021

\author{
Ana Luísa do Nascimento Aquino \\ ORCID: https://orcid.org/0000-0002-9219-939X \\ Centro Superior de Ensino e Pesquisa de Machado, Brasil \\ E-mail: analuisa.aquino@yahoo.com.br \\ Roberta de Oliveira e Silva \\ ORCID: https://orcid.org/ 0000-0002-9243-1478 \\ Universidade Federal de Alfenas, Brasil \\ E-mail: robs1006oliveira@gmail.com
}

\begin{abstract}
Resumo
Em 30 de janeiro de 2020, a Organização Mundial da Saúde (OMS) declarou o surto do COVID-19 uma emergência de saúde pública e, em 11 de março, a epidemia foi atualizado para pandemia. Sem muita cerimônia, a COVID-19 rompeu com a nossa conhecida rotina, alterando nosso cotidiano. Neste novo cenário, incertezas e inseguranças foram acionadas ou intensificadas. Com o avanço da tecnologia, as formas de percepção e interação com o mundo mudaram e a crise ocasionada pela pandemia trouxe consigo uma revolução digital, já que o isolamento social se tornou um fator essencial no enfrentamento da disseminação da doença, com regras rígidas de bloqueio e distanciamento social em muitos países. Frente a este cenário o objetivo deste trabalho foi avaliar a qualidade de vida de cafeicultores da cooperativa COOPFAM da cidade de Poço Fundo-MG através do instrumento SF-36 via questionário online durante a pandemia do COVID-19. O SF-36 é um questionário multidimensional formado por 36 itens, englobados em 8 escalas ou componentes: capacidade funcional, aspectos físicos, dor, estado geral de saúde, vitalidade, aspectos sociais, aspectos emocionais, saúde mental e mais uma questão de avaliação comparativa entre as condições de saúde atual e a de um ano atrás. Avalia tanto os aspectos negativos da saúde (doença ou enfermidade), como os aspectos positivos (bem-estar).
\end{abstract}

Palavras-chave: Qualidade de vida; Inquéritos e questionários; Saúde da população rural.

\begin{abstract}
On January 30, 2020, the World Health Organization (WHO) declared the COVID-19 outbreak a public health emergency, and on March 11, the epidemic was updated to a pandemic. Without much ceremony, COVID-19 broke with our familiar routine, changing our daily lives. In this new scenario, uncertainties and insecurities were triggered or intensified. With the advancement of technology, the forms of perception and interaction with the world have changed and the crisis caused by the pandemic has brought with it a digital revolution, as social isolation has become an essential factor in combating the spread of the disease, with strict blocking rules and social distance in many countries. In view of this scenario, the objective of this study was to evaluate the quality of life of coffee growers in the COOPFAM cooperative in the city of Poço Fundo-MG through the SF-36 instrument via an online questionnaire during the COVID-19 pandemic. The SF-36 is a multidimensional questionnaire consisting of 36 items, encompassed in 8 scales or components: functional capacity, physical aspects, pain, general health, vitality, social aspects, emotional aspects, mental health and one more comparative evaluation question between current health conditions and that of a year ago. It assesses both the negative aspects of health (disease or illness) and the positive aspects (well-being).
\end{abstract} Keywords: Quality of life; Surveys and questionnaires; Rural population health. 


\begin{abstract}
Resumen
El 30 de enero de 2020, la Organización Mundial de la Salud (OMS) declaró que el brote de COVID-19 era una emergencia de salud pública y el 11 de marzo la epidemia se actualizó a pandemia. Sin mucha ceremonia, COVID-19 rompió con nuestra rutina familiar, cambiando nuestra vida diaria. En este nuevo escenario, se dispararon o intensificaron las incertidumbres e inseguridades. Con el avance de la tecnología, las formas de percepción e interacción con el mundo han cambiado y la crisis provocada por la pandemia ha traído consigo una revolución digital, ya que el aislamiento social se ha convertido en un factor fundamental para combatir la propagación de la enfermedad, con estrictas reglas de bloqueo y distancia social en muchos países. Ante este escenario, el objetivo de este estudio fue evaluar la calidad de vida de los caficultores de la cooperativa COOPFAM en la ciudad de Poço Fundo-MG a través del instrumento SF36 a través de un cuestionario en línea durante la pandemia COVID-19. El SF-36 es un cuestionario multidimensional que consta de 36 ítems, englobados en 8 escalas o componentes: capacidad funcional, aspectos físicos, dolor, salud general, vitalidad, aspectos sociales, aspectos emocionales, salud mental y una pregunta más de evaluación comparativa entre la salud actual condiciones y la de hace un año. Evalúa tanto los aspectos negativos de la salud (enfermedad o dolencia) como los aspectos positivos (bienestar).
\end{abstract}

Palabras clave: Calidad de vida; Encuestas y cuestionarios; Salud de la población rural.

\title{
1. Introdução
}

O SARS-CoV-2 surgiu recentemente, tornando-se uma ameaça global, afetando diretamente todos os seres humanos devido à sua morbidade e mortalidade e indiretamente, devido ao enorme impacto econômico e psicológico produzido pelo isolamento social (Romano, et al., 2020).

Os coronavírus causam infecções respiratórias e intestinais em humanos e animais; sendo que a maioria das infecções por coronavírus em humanos são causadas por espécies de baixa patogenicidade, levando ao desenvolvimento de sintomas do resfriado comum, no entanto, podem eventualmente levar a infecções graves em grupos de risco, idosos e crianças (Ministério da Saúde, 2020).

Em 30 de janeiro de 2020, a Organização Mundial da Saúde (OMS) declarou o surto do COVID-19 (Doença Coronavírus 2019) uma emergência de saúde pública e, em 11 de março, a epidemia foi atualizado para pandemia (World Health Organization, 2020).

Sem aviso prévio, a forma de estudar, trabalhar e viver foi alterada (Moretti, et al., 2020). A pandemia de COVID-19 trouxe mudanças drásticas de rotina para a população mundial. O isolamento social, uma das práticas recomendadas para conter o avanço da doença, pode levar ao desenvolvimento de problemas diversos (Reis, et al., 2020). A crescente preocupação com questões relacionadas à qualidade de vida vem de um movimento dentro das ciências humanas e biológicas no sentido de valorizar parâmetros mais amplos que o controle de sintomas, a diminuição da mortalidade ou o aumento da expectativa de vida (Pereira, et al., 2012).

A qualidade de vida das pessoas em meio a pandemias pode ser afetada, tanto em indivíduos saudáveis que precisam permanecer em quarentena e distanciamento social bem como em infectados isolados. (Oliveira et al., 2020)

Em março de 2020, o Ministério da Saúde divulgou a Portaria nº 467, em caráter excepcional e temporário, que regulamenta as ações de Telemedicina, permitindo o atendimento pré-clínico, de suporte assistencial, monitoramento e diagnóstico, por meio de tecnologia da informação e comunicação, no âmbito do Sistema Único de Saúde (SUS), bem como na saúde suplementar e privada (Ministério da Saúde, 2020).

$\mathrm{O}$ crescente uso da internet em todas as faixas etárias, tem instigado os investigadores a desenvolverem questionários virtuais como um método alternativo para a obtenção de respostas em pesquisas científicas (Faleiros, et al., 2016).

O uso de ambientes virtuais para a realização de pesquisas na área de saúde representa uma possibilidade econômica, com maior velocidade de informação e produção científica, capaz de ultrapassar barreiras como idiomas, viabilizando a 
realização de estudos comparativos multicêntricos internacionais, sendo assim, as pesquisas pela internet proporcionam maior praticidade e comodidade aos participantes, o que pode gerar um maior número de participações (Faleiros, et al., 2016).

A COVID-19 avança em todos os continentes, em diferentes culturas e nacionalidades. Impõe necessidades de contenção e isolamento de comunidades e pessoas para minimizar o crescimento exponencial do número de pessoas infectadas (Cruz, et al., 2020).

O município de Poço Fundo destaca-se pelo grande número de agricultores familiares (Dourado, 2017), onde se encontra a Cooperativa dos Agricultores Familiares de Poço Fundo e Região (COOPFAM), que é referência em agricultura orgânica, solidária e agroecológica (Borges, et al., 2013).

Em se tratando de cafeicultura brasileira, o estado de Minas Gerais destaca-se como o maior produtor e exportador, uma vez que possui aparato tecnológico e logístico que facilitam o desenvolvimento dessa atividade agrícola (Do Vale, et al., 2014).

Em termos de produção a mesorregião Sul/Sudeste é a que tem no café a principal atividade econômica em decorrência do grande número de produtores (Do Vale, et al., 2014). Poço Fundo se destaca por ser o município da região com maior presença de agricultores familiares (Dourado, 2017), sendo que o cultivo de café representa 48\% do total da área cultivada no município (Dourado, 2017).

A COOPFAM não busca o desenvolvimento apenas em benefício de seus cooperados, mas vem constantemente contribuindo para alavancar a cafeicultura do município e região, tendo como meta o aumento da qualidade de vida dos associados e da comunidade (Cavino, 2012). Segundo o presidente da COOPFAM, em 2017 a cooperativa possuía 439 famílias de pequenos cafeicultores associados, hoje conta com aproximadamente 560 cooperados e desenvolve diversos programas de incentivo à produção de café orgânico (Dourado, 2017).

Devido à pandemia do COVID-19 declarada como emergência em saúde (World Health Organization, 2020), este trabalho foi realizado via questionário online resguardando aos pesquisadores e voluntários do contato social e prevenindo possíveis contaminações.

Diante deste cenário, o objetivo deste trabalho foi avaliar a qualidade de vida dos cafeicultores da cidade de Poço Fundo-MG através do instrumento SF-36 via questionário online durante a pandemia do COVID-19.

\section{Metodologia}

Trata-se de um estudo transversal, quantitativo (Dalfovo, 2008), com aplicação do questionário SF-36. O estudo foi realizado via questionário online em cafeicultores voluntários participantes de um grupo de WhatsApp da empresa COOPFAM de Poço Fundo-MG. O presente estudo foi aprovado pelo Comitê de Ética e Pesquisa do Centro Superior de Ensino e Pesquisa de Machado (CESEP) sob o número CAAE: 40818820.9.0000.5490. Após aprovação, todos os voluntários foram esclarecidos quanto aos objetivos, à importância das atividades desenvolvidas e os possíveis resultados. Adicionalmente, via WhatsApp concordaram ou não em participar da pesquisa, pois o consentimento será uma das perguntas do questionário online.

Foram incluídos indivíduos cafeicultores e participantes do grupo de WhatsApp da empresa COOPFAM, com idade acima de 18 anos, de ambos os sexos e que concordaram em participar da pesquisa através da pergunta sobre seu consentimento. Foram excluídos indivíduos que não eram cafeicultores ou que não participavam do referido grupo de WhatsApp, e, também indivíduos que não compreenderam a leitura e interpretação do questionário. 


\subsection{Aplicação do questionário SF-36}

Para avaliar a qualidade de vida foi utilizado o questionário SF-36 (Short-Form Health Survey). O SF-36 é um instrumento do tipo genérico criado por Ware e Sherbourne, originalmente na língua inglesa. No Brasil, teve sua tradução e validação cultural realizada por CICONELLI e colaboradores em 1999 (Pereira, et al., 2012).

É composto por 36 itens, que avaliam as seguintes dimensões: capacidade funcional (desempenho das atividades diárias, como capacidade de cuidar de si, vestir-se, tomar banho e subir escadas); aspectos físicos (impacto da saúde física no desempenho das atividades diárias e ou profissionais); dor (nível de dor e o impacto no desempenho das atividades diárias e ou profissionais); estado geral de saúde (percepção subjetiva do estado geral de saúde);vitalidade (percepção subjetiva do estado de saúde); aspectos sociais (reflexo da condição de saúde física nas atividades sociais); aspectos emocionais (reflexo das condições emocionais no desempenho das atividades diárias e ou profissionais) e saúde mental (escala de humor e bem-estar) (Castro, et al., 2003).

A avaliação dos resultados foi feita mediante a atribuição de escores para cada questão, os quais foram transformados numa escala de zero a 100, onde zero corresponde a uma pior qualidade de vida e 100 a uma melhor qualidade de vida. Cada dimensão foi analisada separadamente. (Adorno \& Brasil-Neto, 2013).

\subsection{Análise estatística}

A análise estatística foi feita através do programa estatístico SPSS versão 2.0 (SPSS Inc., Chicago, IL, USA). A análise descritiva foi apresentada em média e desvio padrão (DP). O teste de normalidade para as variáveis estudadas indicou distribuição normal dos dados pelo teste de Kolmogorov-Smirnov, o que permitiu a utilização de teste paramétrico para os dados. Foi utilizado nos domínios do questionário SF-36, e o coeficiente de correlação de Pearson classificando a correlação em perfeita $(r=1)$, forte $(r>0,75)$, moderada $(r>0,5)$, fraca $(r<0,5)$ e inexistente $(r=0)$. O nível de significância para o teste foi de $5 \%$.

\section{Resultados}

As características demográficas estão apresentadas na Tabela 1. Dos 65 voluntários entrevistados, 17 (26,15\%) eram do sexo feminino e $48(73,84 \%)$ eram do sexo masculino. A prevalência do sexo masculino pode ser justificada devido a pesquisa ser respondida via WhatsApp no telefone do cafeicultor cooperado. A idade média da amostra estudada foi de 45,29 anos (DP 11,58). Todos os voluntários foram capazes de responder o questionário de forma completa e não houve desistência durante a participação.

Tabela 1 - Características demográficas.

\begin{tabular}{ll}
\hline $\mathbf{N}$ & $\mathbf{6 5}$ \\
Feminino (\%) & $\mathbf{1 7}(\mathbf{2 6 , 1 5 \% )}$ \\
Masculino (\%) & $\mathbf{4 8}(\mathbf{7 3 , 8 4 \% )}$ \\
\hline Idade (média (DP) & $45,29(11,58)$ \\
\hline
\end{tabular}

Estatística descritiva (SPSS 2.0). Fonte: Autores.

A Tabela 2 mostra os valores obtidos de média e desvio padrão para cada domínio do questionário SF-36. Cada domínio apresenta um escore final de zero a 100, no qual zero corresponde ao pior estado geral de saúde e 100, ao melhor estado de saúde. 
Tabela 2 - valores obtidos para cada domínio do questionário SF-36.

\begin{tabular}{lll}
\hline DOMÍNIOS & MÉDIA & DESVIO PADRÃO \\
\hline Capacidade funcional & 84,846 & 19,079 \\
Aspectos físicos & 66,923 & 39,555 \\
Dor & 57,369 & 27,529 \\
Estado geral da saúde & 60,015 & 10,191 \\
Vitalidade & 60,461 & 15,730 \\
Aspectos sociais & 74,807 & 24,053 \\
Aspectos emocionais & 72,307 & 38,894 \\
Saúde mental & 63,384 & 16,143 \\
\hline
\end{tabular}

Estatística descritiva (SPSS 2.0). Fonte: Autores.

A Tabela 3 apresenta a correlação entre os domínios do questionário SF-36. O domínio capacidade funcional relacionado aos domínios aspectos físicos, dor, estado geral da saúde, vitalidade, aspectos sociais e aspectos emocionais apresentam significância estatística, porém com correlação de Pearson linear positiva fraca. O domínio aspectos físicos apresenta significância estatística com todos os outros domínios e correlação de Pearson linear positiva moderada com os domínios dor e aspectos emocionais e, correlação de Pearson linear positiva fraca com os demais domínios. O domínio dor apresenta significância estatística com todos os outros domínios e correlação de Pearson linear positiva moderada com os domínios capacidade funcional, aspectos físicos, aspectos sociais e aspectos emocionais e, correlação de Pearson linear positiva fraca com os demais domínios. O domínio estado geral da saúde não apresenta significância estatística apenas com os domínios aspectos sociais e aspectos emocionais, porém apresenta correlação de Pearson linear positiva fraca com todos os domínios. O domínio vitalidade apresenta significância estatística com todos os outros domínios e correlação de Pearson linear positiva moderada apenas com o domínio saúde mental. O domínio aspectos sociais não apresenta significância estatística com os domínios capacidade funcional e estado geral da saúde, mas apresenta correlação de Pearson linear positiva moderada com os domínios dor e aspectos emocionais. O domínio aspectos emocionais apresenta significância estatística com todos os outros domínios e correlação de Pearson linear positiva moderada com os domínios aspectos físicos, dor, aspectos sociais e saúde mental. O domínio saúde mental não apresenta significância estatística com o domínio capacidade funcional, e apresenta correlação de Pearson linear positiva moderada somente com os domínios vitalidade e aspectos emocionais.

Tabela 3 - Correlação entre os domínios do questionário SF-36.

\begin{tabular}{|c|c|c|c|c|c|c|c|c|}
\hline & $\begin{array}{l}\text { Capacidade } \\
\text { funcional }\end{array}$ & $\begin{array}{l}\text { Aspectos } \\
\text { físicos }\end{array}$ & Dor & $\begin{array}{l}\text { Estado } \\
\text { geral da } \\
\text { saúde }\end{array}$ & Vitalidade & $\begin{array}{l}\text { Aspectos } \\
\text { sociais }\end{array}$ & $\begin{array}{l}\text { Aspectos } \\
\text { emocionais }\end{array}$ & $\begin{array}{l}\text { Saúde } \\
\text { mental }\end{array}$ \\
\hline $\begin{array}{l}\text { Capacidade } \\
\text { funcional }\end{array}$ & $\mathrm{p}=1$ & $\begin{array}{l}\mathrm{r}=0,379 \\
\mathrm{p}=0,002\end{array}$ & $\begin{array}{l}\mathrm{r}=0,508 \\
\mathrm{p}=0,00\end{array}$ & $\begin{array}{l}\mathrm{r}=0,288 \\
\mathrm{p}=0,020\end{array}$ & $\begin{array}{l}\mathrm{r}=0,336 \\
\mathrm{p}=0,006\end{array}$ & $\begin{array}{l}\mathrm{r}=0,236 \\
\mathrm{p}=0,058\end{array}$ & $\begin{array}{l}\mathrm{r}=0,303 \\
\mathrm{p}=0,014\end{array}$ & $\begin{array}{l}\mathrm{r}=0,097 \\
\mathrm{p}=0,442\end{array}$ \\
\hline $\begin{array}{l}\text { Aspectos } \\
\text { físicos }\end{array}$ & $\begin{array}{l}r=0,379 \\
p=0,002\end{array}$ & $\mathrm{p}=1$ & $\begin{array}{l}r=0,720 \\
p=0,000\end{array}$ & $\begin{array}{l}r=0,472 \\
p=0,000\end{array}$ & $\begin{array}{l}\mathrm{r}=0,307 \\
\mathrm{p}=0,013\end{array}$ & $\begin{array}{l}r=0,486 \\
p=0,000\end{array}$ & $\begin{array}{l}r=0,690 \\
p=0,000\end{array}$ & $\begin{array}{l}r=0,322 \\
p=0,009\end{array}$ \\
\hline Dor & $\begin{array}{l}r=0,508 \\
p=0,000\end{array}$ & $\begin{array}{l}r=0,720 \\
p=0,000\end{array}$ & $\mathrm{p}=1$ & $\begin{array}{l}r=0,385 \\
p=0,002\end{array}$ & $\begin{array}{l}r=0,458 \\
p=0,000\end{array}$ & $\begin{array}{l}r=0,626 \\
p=0,000\end{array}$ & $\begin{array}{l}r=0,617 \\
p=0,000\end{array}$ & $\begin{array}{l}\mathrm{r}=0,383 \\
\mathrm{p}=0,002\end{array}$ \\
\hline $\begin{array}{l}\text { Estado geral } \\
\text { da saúde }\end{array}$ & $\begin{array}{l}r=0,288 \\
p=0,020\end{array}$ & $\begin{array}{l}r=0,472 \\
p=0,000\end{array}$ & $\begin{array}{l}r=0,385 \\
p=0,002\end{array}$ & $\mathrm{p}=1$ & $\begin{array}{l}r=0,364 \\
p=0,003\end{array}$ & $\begin{array}{l}r=0,223 \\
p=0,074\end{array}$ & $\begin{array}{l}r=0,239 \\
p=0,055\end{array}$ & $\begin{array}{l}\mathrm{r}=0,375 \\
\mathrm{p}=0,002\end{array}$ \\
\hline Vitalidade & $\begin{array}{l}r=0,336 \\
p=0,006\end{array}$ & $\begin{array}{l}r=0,307 \\
p=0,013\end{array}$ & $\begin{array}{l}r=0,458 \\
p=0,000\end{array}$ & $\begin{array}{l}r=0,364 \\
p=0,003\end{array}$ & $\mathrm{p}=1$ & $\begin{array}{l}r=0,429 \\
p=0,000\end{array}$ & $\begin{array}{l}r=0,353 \\
p=0,004\end{array}$ & $\begin{array}{l}\mathrm{r}=0,685 \\
\mathrm{p}=0,000\end{array}$ \\
\hline $\begin{array}{l}\text { Aspectos } \\
\text { sociais }\end{array}$ & $\begin{array}{l}r=0,236 \\
p=0,058\end{array}$ & $\begin{array}{l}r=0,486 \\
p=0,000\end{array}$ & $\begin{array}{l}r=0,626 \\
p=0,000\end{array}$ & $\begin{array}{l}r=0,223 \\
p=0,074\end{array}$ & $\begin{array}{l}r=0,429 \\
p=0,000\end{array}$ & $\mathrm{p}=1$ & $\begin{array}{l}r=0,579 \\
p=0,000\end{array}$ & $\begin{array}{l}r=0,466 \\
p=0,000\end{array}$ \\
\hline $\begin{array}{l}\text { Aspectos } \\
\text { emocionais }\end{array}$ & $\begin{array}{l}r=0,303 \\
p=0,014\end{array}$ & $\begin{array}{l}r=0,690 \\
p=0,000\end{array}$ & $\begin{array}{l}r=0,617 \\
p=0,000\end{array}$ & $\begin{array}{l}\mathrm{r}=0,239 \\
\mathrm{p}=0,055\end{array}$ & $\begin{array}{l}r=0,353 \\
p=0,004\end{array}$ & $\begin{array}{l}r=0,579 \\
p=0,000\end{array}$ & $\mathrm{p}=1$ & $\begin{array}{l}\mathrm{r}=0,527 \\
\mathrm{p}=0,000\end{array}$ \\
\hline Saúde mental & $\begin{array}{l}r=0,097 \\
p=0,442\end{array}$ & $\begin{array}{l}\mathrm{r}=0,322 \\
\mathrm{p}=0,009\end{array}$ & $\begin{array}{l}r=0,383 \\
p=0,002\end{array}$ & $\begin{array}{l}r=0,375 \\
p=0,002\end{array}$ & $\begin{array}{l}r=0,685 \\
p=0,000\end{array}$ & $\begin{array}{l}r=0,466 \\
p=0,000\end{array}$ & $\begin{array}{l}r=0,527 \\
p=0,000\end{array}$ & $\mathrm{p}=1$ \\
\hline
\end{tabular}

Correlação de Pearson(r). $r=1$ perfeita, $r>0,75$ forte, $r>0,5$ moderada, $r<0,5$ fraca, $r=0$ inexistente. $p<0,05$. Fonte: Autores. 


\section{Discussão}

Dos 560 cooperados, apenas 65 responderam o questionário, ou seja 11,6\% da amostra total. Identificamos alguns motivos para a baixa adesão como: pandemia, período de colheita ou adubação do café, e falta de incentivo e informação. Diante disso não podemos descrever o perfil dos cooperados mas pode-se ter uma amostra de como está a qualidade de vida do cafeicultor em plena pandemia.

O questionário SF-36 é um instrumento de fácil aplicabilidade que permite avaliar a qualidade de vida através de 8 domínios: capacidade funcional, aspectos físicos, dor, estado geral da saúde, vitalidade, aspectos sociais, aspectos emocionais e saúde mental. O objetivo deste estudo foi avaliar a qualidade de vida de voluntários saudáveis através do instrumento SF-36 via questionário online durante a pandemia do COVID-19.

Entre os domínios do questionário SF-36, as médias mais altas foram para capacidade funcional, aspectos sociais e aspectos emocionais respectivamente e as mais baixas foram para dor e estado geral da saúde. A dor é o domínio que apresenta maior correlação positiva com os outros domínios como capacidade funcional, aspectos físicos, aspectos sociais e aspectos emocionais. Sendo este domínio de grande influência para a mensuração da qualidade de vida, pois afeta a maior parte dos outros domínios. O que nos remete a dura jornada de trabalho do produtor rural e como a mesma compromete sua qualidade de vida, dados semelhantes ao estudo de Moreira et al. (2015) onde os trabalhadores com ocupação agrícola apresentaram maior prevalência de morbidades como dores na coluna ou costas, artrite e reumatismo.

Os resultados nos permitem concluir que, como a média dos escores estão acima de 50, os participantes demostram estar acima da média de qualidade de vida esperada, sendo que a participação do sexo masculino foi relativamente maior do que a do sexo feminino, dados semelhantes aos encontrados por Zhang e Ma (2020), Guirado et al. (2021) e Silva et al. (2021), que concluíram que a pandemia do COVID-19 não causou grandes impactos na qualidade de vida de suas amostras.

\section{Considerações Finais}

Diante do exposto, verifica-se que a pandemia não afetou expressivamente a qualidade de vida dos cafeicultores da cooperativa COOPFAM da cidade de Poço Fundo-MG.

Devido a baixa adesão dos cooperados são necessários mais estudos com uma maior representatividade de entrevistados para uma melhor comparação de resultados.

\section{Agradecimentos}

Nossos sinceros agradecimentos ao Programa de Pós Graduação em Biociências Aplicadas à Saúde da Universidade Federal de Alfenas (UNIFAL), ao Professor Doutor Leonardo César Carvalho (UNIFAL) pelos ensinamentos e ao Centro Superior de Ensino e Pesquisa de Machado/MG (CESEP).

\section{Referências}

Adorno, M. L. G. R. \& Brasil-Neto, J. P. (2013) Avaliação da qualidade de vida com o instrumento SF-36 em lombalgia crônica. Acta Ortop Bras, 21(4): 2027.

Almeida Júnior, S. et al. (2020). COVID-19 e a infecção por SARS-CoV-2 em um panorama geral. Brazilian Journal of Health Review, 3(2): 3508-3522.

Borges, D. A. H. et al. (2013) Análise da participação em uma organização produtora de café orgânico no município de Poço Fundo no sul de Minas Gerais. VIII Simpósio de Pesquisa dos Cafés do Brasil, Salvador.

Castro, M. et al. (2003) Qualidade de vida de pacientes com insuficiência renal crônica em hemodiálise avaliada através do instrumento genérico SF-36. Rev Assoc Med Bras, 49(3): 245-9.

Cavino, V. C. O. (2013) A experiência de comércio justo de agricultores familiares da cooperativa COOPFAM de Poço Fundo (MG): identificando os obstáculos e as potencialidades da transição para a agricultura de base ecológica. UFSCar, São Carlos. 
Research, Society and Development, v. 10, n. 16, e40101623484, 2021

(CC BY 4.0) | ISSN 2525-3409 | DOI: http://dx.doi.org/10.33448/rsd-v10i16.23484

Ciconelli, R. M. et al. (1999) Tradução para a língua portuguesa e validação do questionário genérico de avaliação de qualidade de vida SF-36 (Brasil SF-36). Rev Bras Reumatol, 39(3):143-50.

Cruz, R. M.et al. (2020). COVID-19: Emergência e Impactos na Saúde e no Trabalho. Revista Psicologia: Organizações \& Trabalho (Rpot), Brasilia, p. 2022 .

Dalfovo, M. S.; Lana, R. A. \& Silveira, A. (2008). Métodos quantitativos e qualitativos: um resgate teórico. Revista Interdisciplinar Científica Aplicada, Blumenau, 2(4), 01- 13.

Do Vale, A. R. et al (2014). A cafeicultura em Minas Gerais: estudo comparativo entre as regiões Triângulo Mineiro/Alto Paranaíba e Sul/Sudoeste. CAMPOTERRITÓRIO: Revista de geografia agrária. Edição especial do XXI ENGA-2012, p. 1-23.

Dourado, N. P. (2017). O fortalecimento da agricultura familiar de base agroecológica como vetor de desenvolvimento territorial: o caso da COOPFAM no município de Poço Fundo - MG. VIII Seminário Internacional sobre Desenvolvimento regional: Territórios, Redes e Desenvolvimento Regional: Perspectivas e Desafios. Santa Cruz do Sul.

Faleiros, F. et al. (2016). Use of virtual questionnaire and dissemination as a data collection strategy in scientific studies. Texto \& Contexto - Enfermagem, 25(4): 25-30.

Guirado, G. M. P. et al. (2021). Avaliação da qualidade de vida de trabalhadores antes e durante a pandemia de COVID-19 por meio do questionário SF-36. Rev. Gest. Sist. Saúde, 10(1): 84-105.

Ministério da Saúde (2020). Secretaria de Atenção Especializada à Saúde (SAES). Departamento de Atenção Hospitalar, Domiciliar e de Urgência (DAHU). Protocolo de manejo clínico para o novo coronavírus (2019-nCoV). <https://portalarquivos2.saude.gov.br/images/pdf/2020/fevereiro/11/protocolo-manejocoronavirus.pdf> Acesso em: 26 set. 2020.

Moreira, J. P. L. (2015). A saúde dos trabalhadores da atividade rural no Brasil. Cad. Saúde Pública, 31(8): 1698-1708.

Moretti, S.A. et al. (2020). Nossas Vidas em Meio à Pandemia da COVID - 19: Incertezas e Medos Sociais. Rev Enfermagem e Saúde Coletiva, 4(2): $32-41$.

Oliveira, A. K. B. et al. (2020). Qualidade de vida e distanciamento social: revisão sistemática da literatura. Research, Society and Development, 9(8).

Pereira, E. F. et al. (2012). Qualidade de vida: abordagens, conceitos e avaliação. Rev. Bras. Educ. Fís. Esporte, 26(2): 241-250.

Reis, S. N. et al. (2020). Pandemic, social isolation and the importance of people-plant interaction. Ornamental Horticulture, 26(3): 399-412.

Romano, C.M. et al. (2020). Past, present, and future of COVID-19: a review. Brazilian Journal of Medical and Biological Research, 53(9): 53-59.

Silva, R. O. et al. (2021). Avaliação da qualidade de vida com o instrumento SF-36 durante a pandemia do COVID-19: Um estudo piloto. Research, Society and Development, 10(9).

World Health Organization (2020). Rolling updates on coronavirus disease (COVID-19). < https://www.who.int/emergencies/diseases/novel-coronavirus2019?gclid=EAIaIQobChMIr9Ks562J6gIVTwmRCh1RbwgjEAAYASAAEgK_V_D_BwE>

Zhang, Y., \& Ma, Z. F. (2020). Impact of the COVID-19 Pandemic on Mental Health and Quality of Life among Local Residents in Liaoning Province, China: A Cross-Sectional Study. International Journal of Environmental Research and Public Health, 17(7): 2381. 